\title{
Three-dimensional cfd analysis to study the thrust and efficiency of a biologically- inspired marine propulsor
}

\author{
Lamas M. I., Assoc. Prof. \\ Rodríguez J. D., Assoc. Prof. \\ Rodríguez C. G., M. Sc. \\ González P. B., Prof. \\ Escola Universitaria Politécnica. \\ Universidade da Coruña
}

\begin{abstract}
Aquatic animals, which are the result of many millions of years of evolutionary optimization, are very quick, efficient, robust, and versatile. Accordingly, biologically-inspired mechanisms which emulate the movement of animals have recently become very popular. For the efficient design of a propulsion system it is very important to analyze the fluid flow in detail. CFD (Computational Fluid Dynamics) has become a powerful technique to understand the phenomena because it gives extensive information about the fluid flow characteristics.

In the present work, a propulsion system consisting of an undulating fin which emulates the fish swimming was built. In order to optimize the mechanism, several undulating configurations were studied using a 3D turbulent CFD model. The thrust, drag, efficiency and hydrodynamic characteristics were analyzed. Furthermore, it was shown that the efficiency and thrust depend strongly on the oscillation frequency, amplitude and wavelength.

In order to validate this CFD model, the numerically obtained thrust was successfully compared with experimental results from the laboratory mechanism.
\end{abstract}

Keywords: fish swimming; marine propulsion; undulating fin; biomimetic; computational fluid dynamics; CFD

\section{INTRODUCTION}

Biomimetic is an emerging field which employs the principles of living organisms to derive man-made mechanisms which are capable of emulating the efficient movement of animals. In the field of marine propulsion, undulating fish-like mechanisms offers many advantages over rotary propellers. What makes the high efficiency and high thrust of the biologically movement is the manner in which it works. Behind the fin, several vortices are formed with a rotation direction compatible with the direction of thrust, producing an efficient jet. On the contrary, a propeller generates a long jet that rotates in the direction perpendicular to the motion, wasting a considerable amount of power that goes into rotating this jet.

Under the same conditions, a fish consumes much less energy to displace than a rigid body because the motion of the fish contributes to a reduced drag force and increased propulsive efficiency by reducing separation and suppressing turbulence (Shen et al., 2003). Recent works have shown that undulating fish movement is very efficient compared to rigid bodies (Barret et al., 1999, Liu and Kawachi, 1999, Triantafyllou, 1995) and there are several researchers interested in fish-like underwater and surface ships. For example, Barret et al. (1999) constructed a fish-like mechanism and studied it on a laboratory in order to compare the undulating fish movement with a rigid wall movement. They concluded that the power required to propel an actively swimming fish-like body is significantly smaller than the power needed to tow the body straight and rigid and they obtained drag reductions up to $70 \%$ for the undulating fish movement. Zhang et al. (2008) investigated the bionic neutral network control method for fish-robots and developed a 2D laminar CFD model to study several amplitude envelopes. Young-hua et al. (2007) designed an environment-friendly propulsion system mimicking undulating fins and developed a two-dimensional CFD method to study the unsteady flow around the fin. They focused its study on studying the amplitude configuration. Bozkurtass et al. (2008) constructed a propulsor for an autonomous underwater vehicle based on the mechanical design and performance of a sunfish pectoral fin, and developed a three-dimensional numerical model. Low (2008) constructed an underwater vehicle using a fin-like mechanism based on a series of connecting linkages which produce undulations similar to those produced by the fin rays. Clark and Smith (2007) built a flexible fin which replicates some features of the pectoral fin of a batoid fish.

Although these studies have revealed many key points of undulating propulsion, much is still unknown regarding the 
behavior of the unsteady fluid flow. Recently, CFD has become a powerful tool to study these phenomena. By solving the Navier-Stokes equations, CFD provides information on the pressure and velocity fields. It is very useful to complement traditional testing and experimentation, providing faster time and lower costs. In this work, an undulating propulsor which emulates the high performance and maneuvering of fish swimming was built. In order to analyze the flow around the fin, a tri-dimensional turbulent CFD model was developed. A previously 2D CFD model was already developed elsewhere (Lamas et al., 2010). In the present paper, the 2D model was extended to the more realistic 3D situation. This numerical model was satisfactory compared with the experimental data obtained from the experimental prototype. Once validated, the CFD analysis was employed to predict how the performance varies with different parameters.

This paper begins with a introduction of the experimental prototype and the kinematics of locomotion. The numerical method and results are then briefly described and contrasted with experimental data. Finally, the conclusions of this work and the areas for future research are presented.

\section{PROBLEM DEFINITION}

\section{Design and kinematics}

The experimental ship is shown in Fig. 1.a. The propulsive fin, which is shown in Fig. 1.b, consists of a flexible surface of $0.52 \mathrm{~m}$ length and $0.2 \mathrm{~m}$ width.

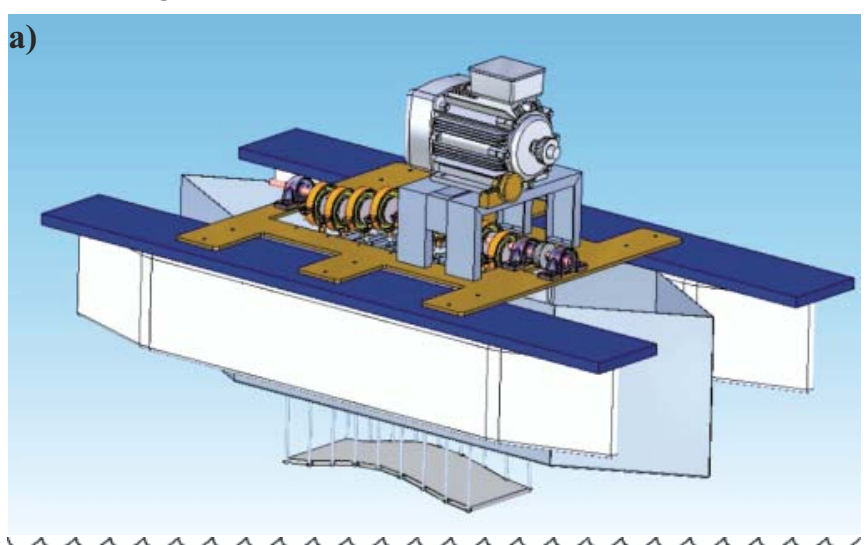

b)

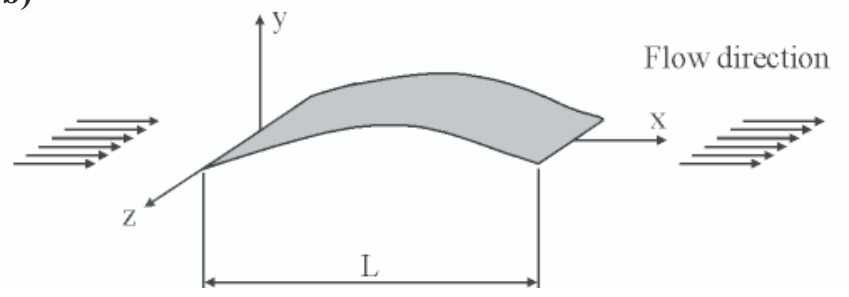

Fig. 1. a) experimental mechanism, b) detail of the fin

Fig. 2 provides the mechanism of the undulating fin, consisting of eight rigid segments, each capable of relative rotation with respect to its neighboring links. The movement of each segment is produced by a crankshaft situated above the fin. The power is supplied by an electric motor and the oscillation frequency is controlled by a variable-frequency drive control.

Every module is attached to a connecting rod on both sides. The position of every segment is defined by the rotation of the crankshaft, which moves the rods, the connecting rods, and finally the segment. In order to investigate what kind of

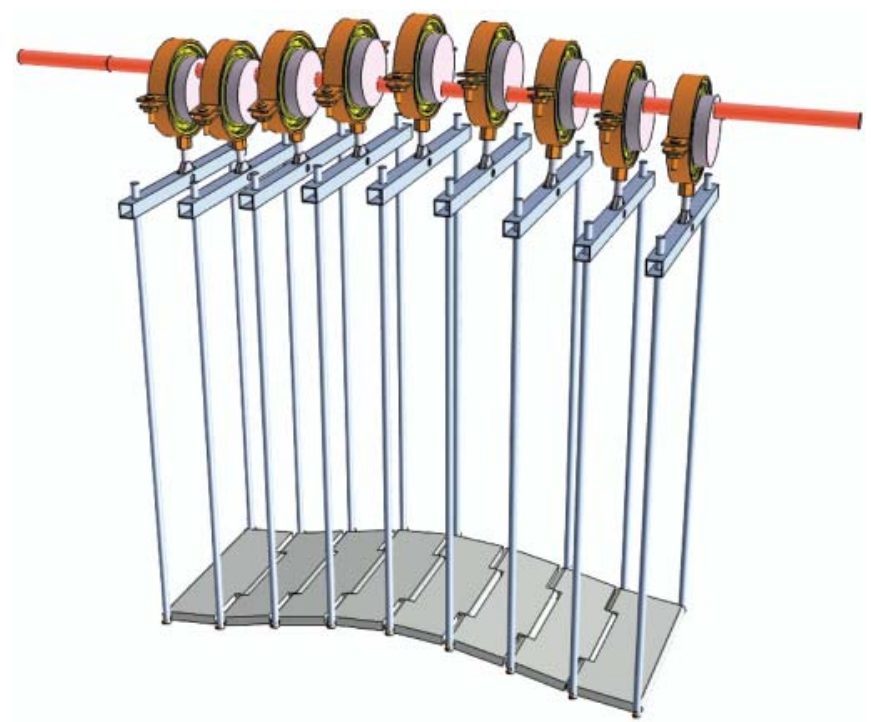

Fig. 2. Detail of the undulating surface and mechanical transmission of movement

amplitude envelope provides the maximum efficiency, the mechanism was designed to accommodate several centimeters peak-to-peak amplitude. More details of the system were given elsewhere (Rodríguez, 2009).

The high thrust and efficiency are not the only advantages of this system. It is also reversible, i.e., it has the same efficiency either operating forward or backward. This makes it ideal for vehicles that require high maneuverability. Other advantage is that the use of several properly positioned fins can minimize vibrations.

\section{Governing equations}

The fluid flow around this mechanism was studied numerically. In order to simplify the model, the flow was considered incompressible and isothermal. The unsteady turbulent flow was simulated by the RANS (Reynolds-averaged Navier-Stokes) equations. In Cartesian tensor form, the mass conservation equation is given by:

$$
\frac{\partial \rho}{\partial t}+\frac{\partial}{\partial x_{i}}\left(\rho u_{i}\right)=0
$$

and the momentum conservation equation:

$$
\begin{gathered}
\frac{\partial}{\partial t}\left(\rho u_{i}\right)+\frac{\partial}{\partial x_{j}}\left(\rho u_{i} u_{j}\right)= \\
=-\frac{\partial p}{\partial x_{i}}+\frac{\partial \tau_{i j}}{\partial x_{j}}+\frac{\partial}{\partial x_{j}}\left(-\rho \overline{u_{i}^{\prime} u_{j}^{\prime}}\right)
\end{gathered}
$$

Since the fluid was treated as Newtonian, the stress tensor components are given by:

$$
\tau_{\mathrm{ij}}=\mu\left(\frac{\partial \mathrm{u}_{\mathrm{i}}}{\partial \mathrm{x}_{\mathrm{j}}}+\frac{\partial \mathrm{u}_{\mathrm{j}}}{\partial \mathrm{x}_{\mathrm{i}}}-\frac{2}{3} \delta_{\mathrm{ij}} \frac{\partial \mathrm{u}_{\mathrm{k}}}{\partial \mathrm{x}_{\mathrm{k}}}\right)
$$

A common method to model the Reynolds stresses, $-\rho \overline{u_{i}^{\prime} u_{j}^{\prime}}$, is the Boussinesq hypothesis to relate the Reynolds stresses to the mean velocity gradients:

$$
-\rho \overline{u_{i}^{\prime} u_{j}^{\prime}}=\mu_{t}\left(\frac{\partial u_{i}}{\partial x_{j}}+\frac{\partial u_{j}}{\partial x_{i}}\right)-\frac{2}{3}\left(\rho k+\mu_{t} \frac{\partial u_{k}}{\partial x_{k}}\right) \delta_{i j}
$$

where $\delta_{\mathrm{ij}}$ is the Kronecker delta $\left(\delta_{\mathrm{ij}}=1\right.$ if $\mathrm{i}=\mathrm{j}$ and $\delta_{\mathrm{ij}}=0$ if $\mathrm{i} \neq \mathrm{j}$ ) which is included to make the formula applicable to the normal Reynolds stresses for which $\mathrm{i}=\mathrm{j}$ (Versteeg and 
Malalasekera, 2007) and $\mu_{t}$ is the turbulent viscosity. Since the turbulent viscosity was modeled by the k- $\varepsilon$ model, two transport equations are added, one for the turbulence kinetic energy $\mathrm{k}$ (eq. 5 ) and a further one for its dissipation rate $\varepsilon$ (eq. 6) respectively. Unfortunately, no single turbulence model is universally accepted. The reasons why the $\mathrm{k}-\varepsilon$ model was applied in this work are that it is robust, computationally economical and reasonably accurate for a wide range of turbulent flows.

$$
\begin{gathered}
\frac{\partial}{\partial \mathrm{t}}(\rho \mathrm{k})+\frac{\partial}{\partial \mathrm{x}_{\mathrm{i}}}\left(\rho \mathrm{ku}_{\mathrm{i}}\right)= \\
=\frac{\partial}{\partial \mathrm{x}_{\mathrm{j}}}\left[\left(\mu+\frac{\mu_{\mathrm{t}}}{\sigma_{\mathrm{k}}}\right) \frac{\partial \mathrm{k}}{\partial \mathrm{x}_{\mathrm{j}}}\right]+\mathrm{G}_{\mathrm{k}}+\mathrm{G}_{\mathrm{b}}-\rho \varepsilon-\mathrm{Y}_{\mathrm{M}} \\
\frac{\partial}{\partial \mathrm{t}}(\rho \varepsilon)+\frac{\partial}{\partial \mathrm{x}_{\mathrm{i}}}\left(\rho \varepsilon \mathrm{u}_{\mathrm{i}}\right)=\frac{\partial}{\partial \mathrm{x}_{\mathrm{j}}}\left[\left(\mu+\frac{\mu_{\mathrm{t}}}{\sigma_{\varepsilon}}\right) \frac{\partial \varepsilon}{\partial \mathrm{x}_{\mathrm{j}}}\right]+ \\
+\mathrm{C}_{1 \varepsilon} \frac{\varepsilon}{\mathrm{k}}\left(\mathrm{G}_{\mathrm{k}}+\mathrm{G}_{3 \varepsilon} \mathrm{G}_{\mathrm{b}}\right)-\mathrm{C}_{2 \varepsilon} \rho \frac{\varepsilon^{2}}{\mathrm{k}}
\end{gathered}
$$

In the above equations, $G_{k}$ represents the generation of turbulence kinetic energy due to the mean velocity gradients; $\mathrm{G}_{\mathrm{b}}$ is the generation of turbulence kinetic energy due to buoyancy; $\mathrm{Y}_{\mathrm{M}}$ represents the contribution of the fluctuating dilatation in compressible turbulence to the overall dissipation rate. $\mathrm{C}_{\mu}, \mathrm{C}_{1 \varepsilon}, \mathrm{C}_{2 \varepsilon}, \mathrm{C}_{3 \varepsilon}, \sigma_{\mathrm{k}}$ and $\sigma_{\varepsilon}$ are constants with the standard $\mathrm{k}-\varepsilon$ default values $\mathrm{C}_{\mu}=0.09 ; \mathrm{C}_{1 \varepsilon}=1.44 ; \mathrm{C}_{2 \varepsilon}=1.92 ; \sigma_{\mathrm{k}}=1.0$ and $\sigma_{\varepsilon}=1.3$. Details are given in (Fluent Inc., 2006).

The turbulent viscosity, $\mu_{\mathrm{t}}$, Is computed by combining $\mathrm{k}$ and $\varepsilon$ as follows:

$$
\mu_{\mathrm{t}}=\rho \mathrm{C}_{\mu} \frac{\mathrm{k}^{2}}{\varepsilon}
$$

\section{NUMERICAL IMPLEMENTATION}

\section{Computational mesh}

In order to implement the movement of the fin, a moving mesh which regenerates with time was employed. The computational domain is shown in Fig. 3, in which the front surface and the internal mesh were omitted for clarity. It was discretized with 600000 tetrahedral elements. The size mesh was refined near the fin and in the wake region to resolve the shed vortices as they travel downstream. Due to the symmetry of the problem, only half of the geometry was modeled.

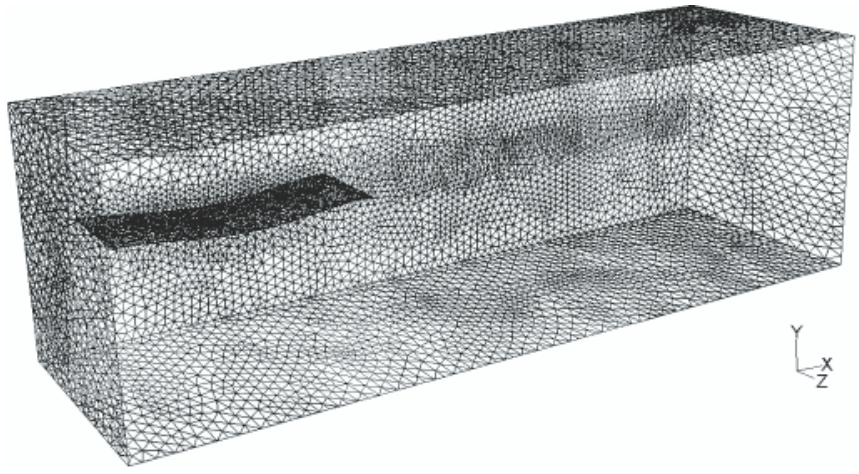

Fig. 3. Computational domain

\section{Boundary Conditions}

The downstream boundary (right surface) was modeled as a pressure outlet and the upstream boundary condition (left surface) was modeled as an uniform velocity inlet corresponding to the free-stream velocity. The fin surface was treated as a no-slip moving wall. The top and bottom sides were modeled as no slip walls and the front surface was treated as a symmetry plane.

\section{Numerical Methodology}

The numerical solution was performed using the commercial software Ansys Fluent 6.3, which is based on the finite volume method. Pressure-velocity coupling was achieved using the PISO algorithm. A second order scheme was used for discretization of the pressure and momentum equations. The time integration was achieved using a first order fully implicit scheme with a constant time step of $0.001 \mathrm{~s}$.

Extensive convergence checks were taken in order to confirm that the computed results are independent of the time step, the grid size and the computational domain size.

\section{CALCULATION OF FORCES AND EFFICIENCY}

As the fin moves through the water, a force is produced along the $\mathrm{x}, \mathrm{y}$ and $\mathrm{z}$ directions. Particularly, a pressure force is caused due to pressure difference and a friction force is caused due to water viscidity. The components of the forces acting on the body, $\mathrm{F}_{\mathrm{x}}, \mathrm{F}_{\mathrm{y}}$ and $\mathrm{F}_{\mathrm{z}}$ can be evaluated by integrating the projection of the pressure and the shear stress in the $\mathrm{x}, \mathrm{y}$ and $\mathrm{z}$ directions respectively. The total component along the $\mathrm{x}$ direction was computed by adding the component $\mathrm{x}$ of the pressure and viscous forces acting on the body as follows:

$$
\mathrm{F}_{\mathrm{x}}=\mathrm{F}_{\mathrm{px}}+\mathrm{F}_{\mathrm{vx}}
$$

where:

$\mathrm{F}_{\mathrm{px}}$ - the pressure force

$\mathrm{F}_{\mathrm{vx}}^{\mathrm{px}}-$ the viscous force in the $\mathrm{x}$ direction.

The pressure force along the $\mathrm{x}$ axis is given by:

$$
\mathrm{F}_{\mathrm{px}}=-\int_{\mathrm{A}} \mathrm{pn}_{\mathrm{x}} \mathrm{dS}
$$

where:

$n_{x}-$ the $x$ component of the unit normal vector on $d S$.

The viscous force along the $\mathrm{x}$ axis is given by:

$$
F_{v x}=\int_{A} \tau_{x j} n_{j} d S
$$

where:

$\tau_{\mathrm{xj}}-$ the viscous stress tensor.

The total power required for the propulsive motion of the fin $\left(\mathrm{P}_{\mathrm{T}}\right)$ consists of two components, $\mathrm{P}_{\mathrm{T}}=\mathrm{P}_{\mathrm{S}}+\mathrm{P}_{\mathrm{U}}$. One is the swimming power $\left(\mathrm{P}_{\mathrm{S}}\right)$ required to produce the vertical oscillations of the fin and the other is the useful power $\left(\mathrm{P}_{U}\right)$, required to thrust. The swimming power was computed as:

$$
\mathrm{P}_{\mathrm{s}}=\int \mathrm{p} \frac{\mathrm{dy_{ \text {wall } }}}{\mathrm{dt}} \mathrm{dS}
$$

where $\mathrm{p}$ is the pressure of the surface of the plate and $\mathrm{dy}_{\text {wall }} / \mathrm{dt}$ refers to the y-component of the velocity at the surface of the fish. For a sinusoidal wavy the motion of the fin and the y-component of the velocity are given by Ec. (12) and (13) respectively.

$$
y=A(x) \cdot \sin \left[2 \pi\left(\frac{x}{\lambda}-\frac{t}{T}\right)\right]
$$




$$
\frac{\mathrm{dy}_{\text {wall }}}{\mathrm{dt}}=-\frac{2 \pi}{\mathrm{T}} \mathrm{A}(\mathrm{x}) \cdot \cos \left[2 \pi\left(\frac{\mathrm{x}}{\lambda}-\frac{\mathrm{t}}{\mathrm{T}}\right)\right]
$$

where:

$\mathrm{A}(\mathrm{x})$ - represents the amplitude,

$\lambda \quad-$ the wavelength,

$\mathrm{T} \quad-$ the period.

The useful power was computed as the time-average thrust multiplied by the free-stream velocity:

$$
\mathrm{P}_{\mathrm{U}}=-\mathrm{F}_{\mathrm{px}} \mathrm{U}_{\infty}
$$

where the minus sign indicates that the velocity of swimming is reverse to that of the stream.

The efficiency was computed as the ratio between the useful power and the total power. Obviously, the efficiency is always less than one because of losses due to wayward vortices and turbulence.

\section{RESULTS AND DISCUSSION Configurations Studied}

The motion of the fin is in the form of a streamwise travelling wave. As the fluid moves along the surface, the undulations from the anterior to the posterior of the fin produce thrust. The amplitude may vary along the length, and its design is very important. For this reason, several CFD analysis were carried out to assess the most suitable configuration. Three cases were studied: amplitude increasing from the anterior part to the posterior (Fig. 4a), constant amplitude (Fig. 4b) and amplitude increasing from the anterior part to the mid part and decreasing towards the posterior (Fig. 4c).

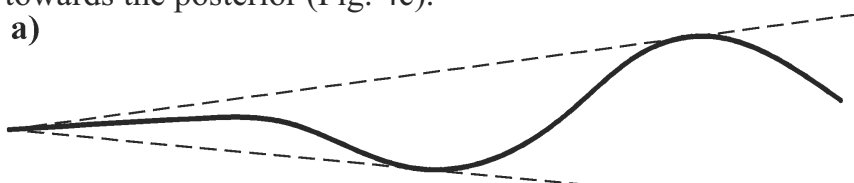

b)

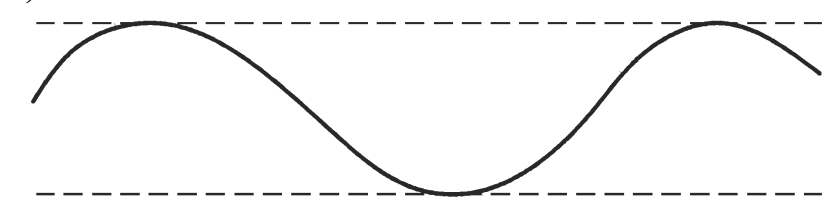

c)

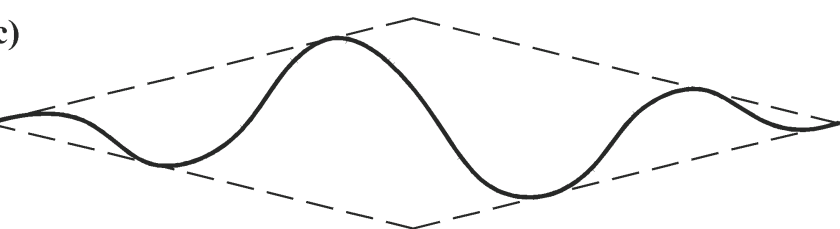

Fig. 4. Amplitude configurations. a) amplitude increasing from the anterior part to the posterior, $\boldsymbol{b})$ constant amplitude, $\boldsymbol{c}$ ) amplitude increasing from

the anterior part to the mid part and decreasing towards the posterior

\section{Hydrodynamic forces and efficiency}

Fig. 5a represents the time history of the instantaneous pressure force, viscous force and average total force along the cycle at $\mathrm{U}_{\infty}=0 ; \mathrm{f}=10 \mathrm{~Hz} ; \mathrm{A}_{\max }=0.02 \mathrm{~m}$ and $\lambda=1 \mathrm{~m}$. The zero free-stream velocity corresponds to a experimental situation in which the ship is tied to a fixed point by means of a rope, Fig. 5b. The configuration studied in Fig. 5a corresponds to (a), i.e., amplitude increasing from the anterior part to the posterior, and the other two amplitude configurations presented a similar tendency, so they are not plotted again.
In order to reach a situation which periodically repeats, i.e., a quasy-steady state, it was necessary to study a long enough interval of time. For the cases studied it was verified that this state is achieved after approximately twenty fin oscillation periods. For this reason, all the results carried out in the present paper correspond to the $20^{\text {th }}$ period of time.
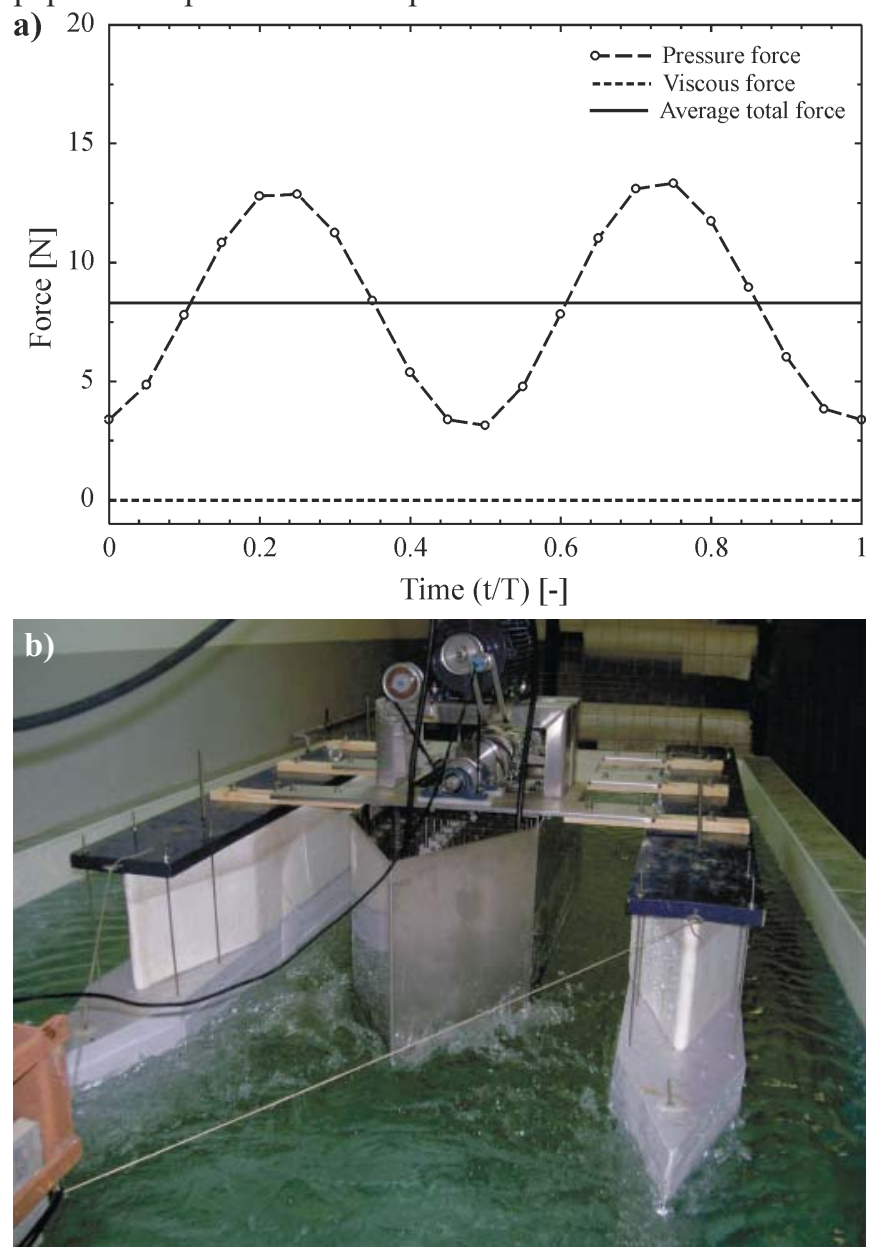

Fig. 5. a) time history of the instantaneous thrust and drag and average total force at $U_{\infty}=0 ; f=10 \mathrm{~Hz} ; A_{\max }=0.02 \mathrm{~m}$ and $\lambda=1 \mathrm{~m}$. Configuration (a), amplitude increasing from the anterior part to the posterior; b) equivalent experimental situation (pulling against a fixed point)

The forces shown in Fig. 5a are the ones that accelerate the fin either forward or backward, depending on its sign. For the above conditions the mechanism is accelerating, i.e., the pressure force is much higher than the viscous force. If the rope is broken and the ship is allowed to displace, after accelerating it tends towards an asymptotic state in which the mean velocity is constant and the net thrust (i.e., sum of pressure and viscous force) is zero. For the parameters of the figure above, this situation is obtained at $\mathrm{U}_{\infty}=5.1 \mathrm{~m} / \mathrm{s}$, and the respective forces are represented in Fig. 6 .

From the figures above, it can be observed that the instantaneous pressure force reaches its maximum value twice in each cycle because of the symmetrical undulating movement. The maximum thrust takes place at the time that the ends of the fin are in the position corresponding to zero amplitude. On the contrary, the worst case occurs at the point where the edges of the fin are in the position corresponding to its maximum amplitude.

The period-average thrust and efficiency of the three cases studied are summarized in Table 1 , in which the negative sign was used to denote the direction opposite to that of the stream. 


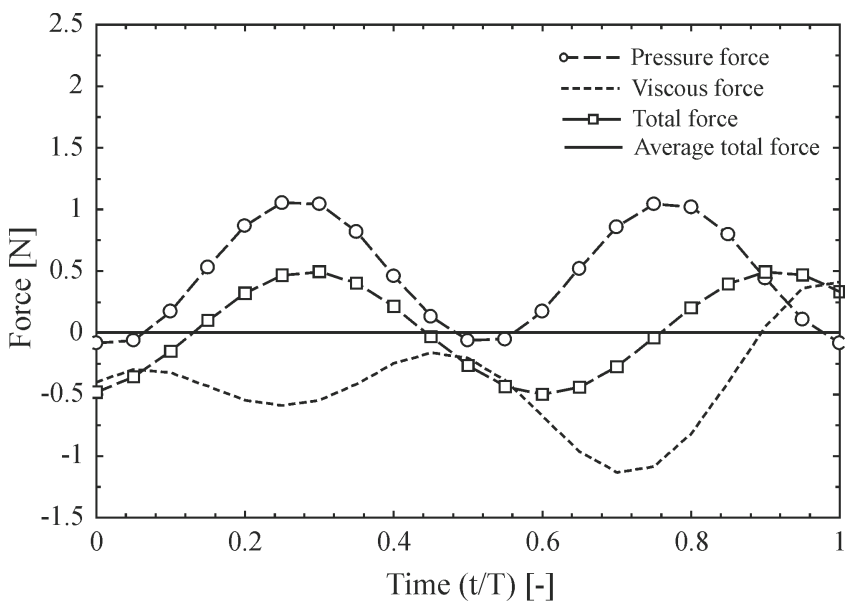

Fig. 6. Time variation of instantaneous thrust and drag and average total force at $U_{\infty}=5.1 \mathrm{~m} / \mathrm{s} ; f=10 \mathrm{~Hz} ; A_{\max }=0.02 \mathrm{~m}$ and $\lambda=1 \mathrm{~m}$. Configuration (a), amplitude increasing from the anterior part to the posterior

Tab. 1. Period-averaged thrust and efficiency for the three fin undulating patterns at $U_{\infty}=5.1 \mathrm{~m} / \mathrm{s} ; f=10 \mathrm{~Hz} ; A_{\max }=0.02 \mathrm{~m}$ and $\lambda=1 \mathrm{~m}$

\begin{tabular}{|c|c|c|}
\hline $\begin{array}{c}\text { Fin undulating } \\
\text { pattern }\end{array}$ & Thrust (N) & Efficiency (\%) \\
\hline Configuration (a) & 0 & 44.4 \\
\hline Configuration (b) & 1.8 & 34.5 \\
\hline Configuration (c) & -2.96 & 22.5 \\
\hline
\end{tabular}

(a) Amplitude increasing from the anterior part to the posterior.

(b) Constant amplitude.

(c) Amplitude increasing from the anterior part to the mid part and decreasing towards the posterior.

From Table 1, it can be seen that the case with amplitude increasing from the anterior part to the mid part and decreasing towards the posterior (configuration c) is the worst configuration because it corresponds to the lowest efficiency and thrust. The case with constant amplitude (configuration b) produces the highest thrust and the one with amplitude increasing from the anterior part to the posterior (configuration a) produces the highest efficiency. This conclusion was expected because the travelling waves with increasing amplitude are a typical form adopted for swimming motion of live fish in nature. The most effective amplitude configuration, case a, will be discussed further in the following sections.

\section{Pressure field}

Fig. 7 shows the pressure distribution in the zone closed to the fin. It corresponds to the pattern with amplitude increasing from the anterior part to the posterior at $\mathrm{U}_{\infty}=5.1 \mathrm{~m} / \mathrm{s} ; \mathrm{f}=10$ $\mathrm{Hz} ; \mathrm{A}_{\max }=0.02 \mathrm{~m}$ and $\lambda=1 \mathrm{~m}$.

The iso-pressure contours show areas of high and low pressures. Behind the fin a high pressure region is created and hence a propulsive jet which produces thrust. As discussed previously, the peak for pressure and viscous forces do not occur simultaneously. The maximum pressure force occurs at instants of approximately maximum amplitude at the tail, when the pressure gradient attached to the body acts to pull the fish upstream, 0.25t/T and $0.75 \mathrm{t} / \mathrm{T}$ in Fig. 7. On the other hand, the minimum viscous force occurs when the undulatory wave has a maximum amplitude at approximately the midpoint of the fin, $0.5 \mathrm{t} / \mathrm{T}$ in Fig. 7, when the pressure gradients are smaller.

From Fig. 7 it can also be seen that a staggered array of reverse von Karman vortices forms behind the fin, which

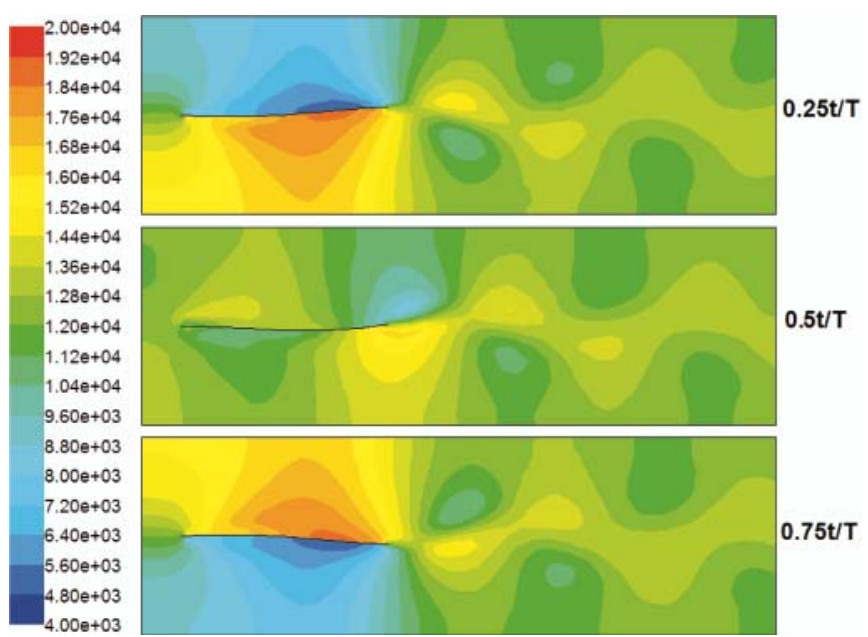

Fig. 7. Pressure field (Pa) at $U_{\infty}=5.1 \mathrm{~m} / \mathrm{s} ; f=10 \mathrm{~Hz} ; A_{\max }=0.02 \mathrm{~m}$ and $\lambda=1 \mathrm{~m}$. Configuration (a), amplitude increasing from the anterior part to the posterior

decay downstream due to viscous dissipation. These vortices are very important because they are closely related to the jet stream which generates an opposite force to thrust the body forward and their optimal formation would increase efficiency noticeable.

\section{Velocity field}

Fig. 8 shows the velocity field of the pattern with amplitude increasing from the anterior part to the posterior at $\mathrm{U}_{\infty}=5.1 \mathrm{~m} / \mathrm{s}$; $\mathrm{f}=10 \mathrm{~Hz} ; \mathrm{A}_{\max }=0.02 \mathrm{~m}$ and $\lambda=1 \mathrm{~m}$. It corresponds to Fig. 7.

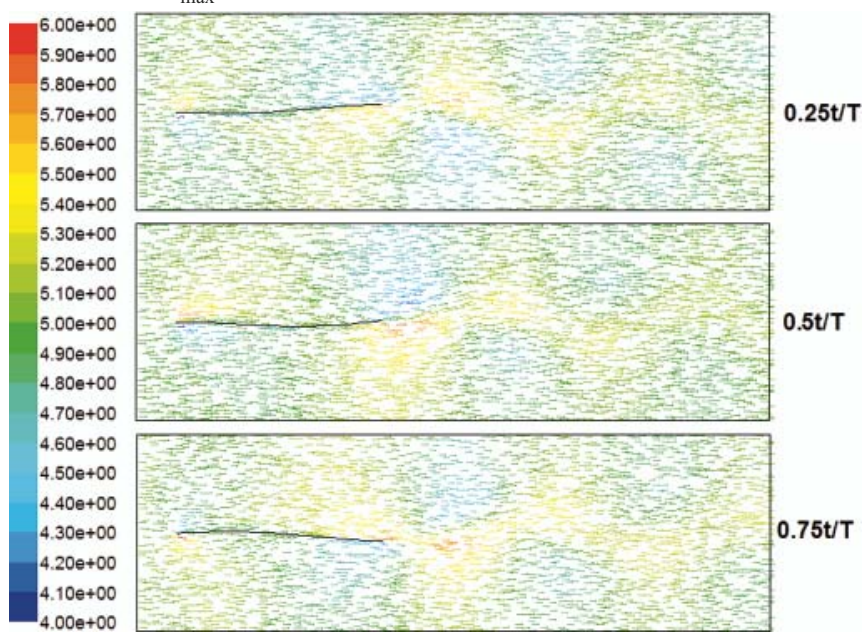

Fig. 8. Velocity field $(\mathrm{m} / \mathrm{s})$ at $U_{\infty}=5.1 \mathrm{~m} / \mathrm{s} ; f=10 \mathrm{~Hz} ; A_{\max }=0.02 \mathrm{~m}$ and $\lambda=1 \mathrm{~m}$. Configuration (a), amplitude increasing from the anterior part to the posterior

In this picture, the downstream-directed jet and the von Karman vortices are appreciated too. The streamwise velocity in this thrust jet is as much as $20 \%$ greater than the free-stream velocity, so the fish increases the momentum of the fluid in the wake.

\section{Influence of the amplitude, frequency and wavelength}

The influence of the maximum amplitude, oscillation frequency and wavelength on the thrust and efficiency was analyzed and represented in Fig. 9. This figure was carried out for the configuration (a), amplitude increasing from the anterior part to the posterior and zero free-stream velocity. The other two configurations presented similar tendencies. 

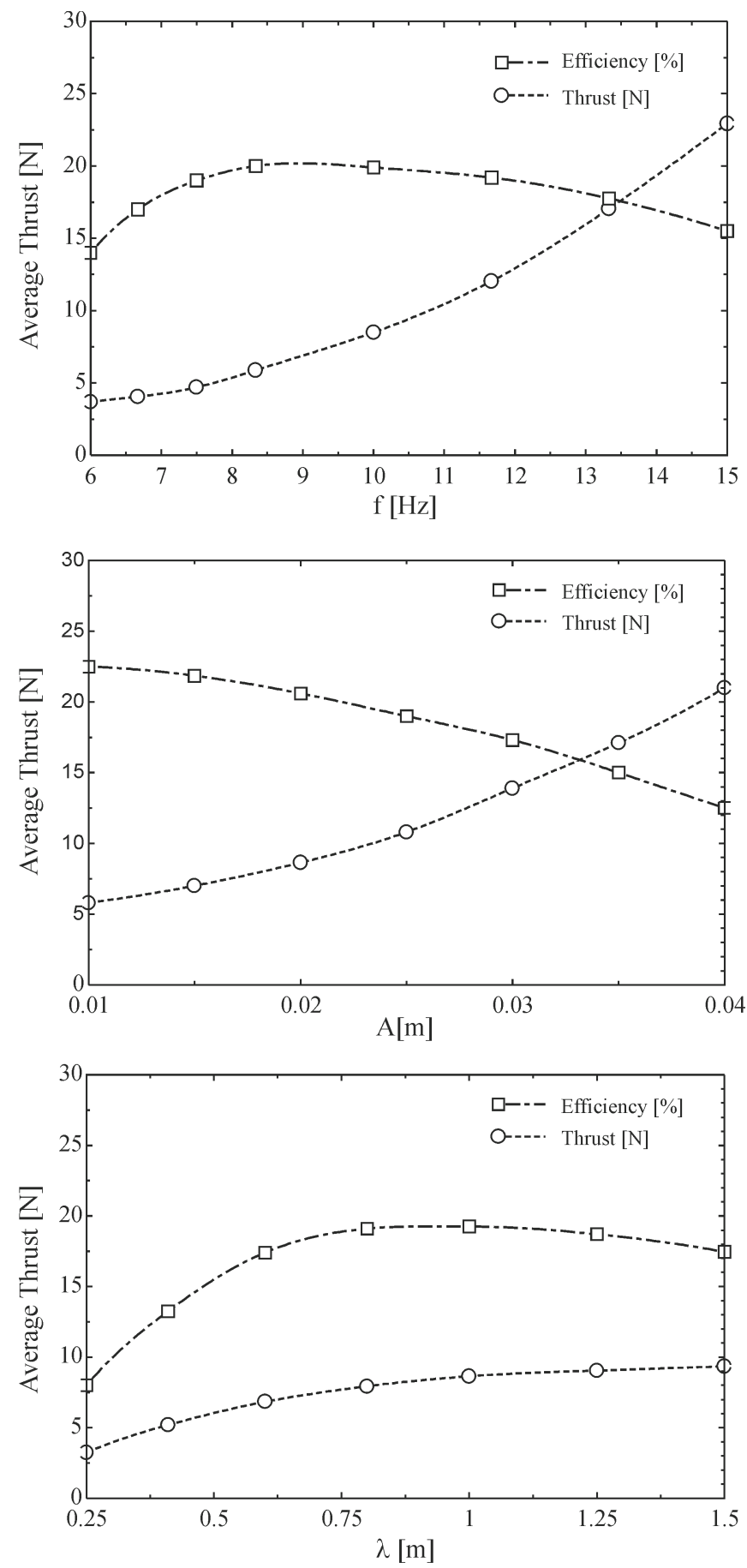

Fig. 9. Variation of the period-average thrust and efficiency with amplitude frequency and wavelength at $U_{\infty}=0 \mathrm{~m} / \mathrm{s}$. Configuration (a), amplitude increasing from the anterior part to the posterior

From the figures above it can be seen that, as expected, the thrust increases when the oscillation frequency increases. As the frequency increases, the efficiency increases rapidly to its maximum and then decreases gradually. Other studies realized in flapping foils and fishes confirmed that the efficiency is maximum in a limited range of frequency. (Triantafyllou, 1995; Liu and Kawachi, 1999; Deng et al., 2005).

In what concerns the maximum amplitude, it can be seen that the thrust increases with increasing amplitude. However, this it at the expense of even larger power cost required and hence less efficiency. On the other hand, at smaller amplitudes the efficiency is higher but it is more difficult to generate an effective thrust.

In what concerns the wavelength, as the wavelength increases, the average thrust at first increases up to a certain maximum value and then decreases, while the efficiency increases monotonically.

From the calculated results, the best option should be a combination of thrust and efficiency. It would depend on every practical case. For example, military applications require high specific power even though the efficiency is not optimal. On the contrary, for civil applications the most important issue is the efficiency.

\section{EXPERIMENTAL RESULTS}

A validation test was carried out in order to assess the adequacy of the computational model developed here. The experimental results were based on tying the ship to a fixed point by means of a rope, Fig. 5 b. Once the prototype was fixed, the force was measured at different oscillation frequencies of the fin. A load cell was used to measure these forces and a variable-frequency drive control was used to control the oscillation frequency of the electrical motor.

Numerically, it was simulated with zero free-stream velocity. Numerical and experimental results for the average thrust force against the frequency are compared in Fig. 10.


Fig. 10. Average force against frequency for numerical and experimental results. a) amplitude increasing from the anterior part to the posterior; b) constant amplitude

From the figure above, it can be seen that the experimental results were in good agreement with numerical ones, which means that this CFD model can accurately predict the physical phenomena.

\section{CONCLUSIONS}

In this work, a fish-like marine propulsor was designed and the fluid around the mechanism was numerically investigated 
by solving the three-dimensional incompressible Navier-Stokes equations. The hydrodynamics of the fluid flow, pressure and velocity fields were obtained. The efficiency and forces (which were decomposed into pressure and viscous forces) were obtained too. The influence of the amplitude envelope was studied, concluding that the configuration with amplitude increasing from the anterior part to the posterior is the most efficient. Several values of the frequency, wavelength and maximum amplitude were analyzed and it was found that the efficiency and thrust depend strongly on these parameters. Particularly, the thrust increases both with the increase of the frequency and the amplitude; the efficiency decreases with increasing the amplitude and as the frequency or the wavelength increases, the efficiency increases to its maximum and then decreases gradually. For this reason, the results obtained in this study provide interesting information on the performance of fish-like mechanisms and demonstrate that a properly design of the fin can be very appropriate for ships and underwater vehicles. The optimal propulsive mechanism would be a combination of the properly thrust or efficiency, depending on every practical application.

While CFD is a very powerful tool, it can not be applied blindly as it has the capability to produce non-physical results due to erroneous modeling. For this reason, the numerically obtained thrust was compared with experimental results from the laboratory mechanism to ensure that the model accurately predicts the physical phenomena.

\section{Acknowledgements}

The authors wish to thank to Xunta de Galicia its financial support through the project "Sistema de propulsión ondulante", PGIDIT06DPI172PR8.

\section{NOMENCLATURE}
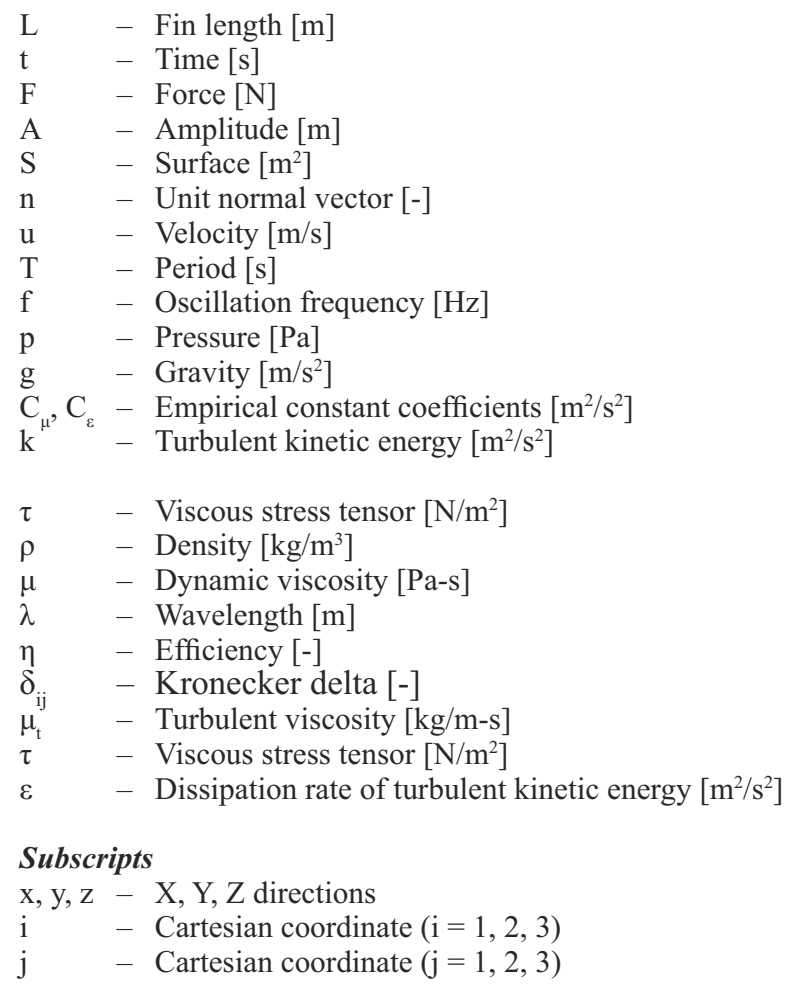

$$
\begin{array}{ll}
\max & - \text { Maximum } \\
\mathrm{p} & - \text { Pressure } \\
\mathrm{V} & - \text { Viscosity } \\
\mathrm{T} & - \text { Total } \\
\mathrm{S} & - \text { Swimming } \\
\mathrm{U} & - \text { Useful } \\
\infty & - \text { Free-stream }
\end{array}
$$

\section{BIBLIOGRAPHY}

1. Shen, L., Zhang, X., Yue, D. K. P., (2003), Turbulent flow over a flexible wall undergoing a streamwise travelling wave motion, Journal of Fluid Mechanics, vol. 484, pp. 197-221.

2. Barret, D. S., Triantafyllou, M. S., Yue, D. K. P., Grosenbaugh, M. A., and Wolfgang, M. J. (1999) Drag reduction in fish-like locomotion. Journal of Fluid Mechanics 392, 183-212.

3. Liu, H., Kawachi, K. (1999) A numerical study of undulatory swimming, Journal of Computational Physics 155, pp. 223-247.

4. Triantafyllou, M. S., Triantafyllou, G. S., (1995). An efficient swimming machine. Scientific American, vol. 272, issue 3, pp. 64-70.

5. Zhang, D., Hu, D., Shen, L., Xie, H., (2008), Design of an artificial bionic neural network to control fish-robot's locomotion, Neurocomputing 71, pp. 648-654.

6. Yong-hua Zhang, Jia, L., Zhang, S., Yang, J., Low, K. H. (2007) Computational research on modular undulating fin for biorobotic underwater propulsor. Journal of Bionic Engineering 4, 25-32.

7. Bozkurttas, M., Tangorra, J., Lauder, G., Mittal, R. (2008) Understanding the hydrodynamics of swimming: from fish fins to flexible propulsors for autonomous underwater vehicles. Advances in Science and Technology 58, 193-202.

8. Low, K. H. (2008) Locomotion simulation and system integration of robotic fish with modular undulating fin. International Journal of Simulation 7 (8), 64-77.

9. Clark, R. P., Smith A. J., (2006) Thrust production and wake structure of a batoid-inspired oscillating fin, Journal of Fluid Mechanics 562, pp. 415-429.

10.Lamas, M. I., Rodríguez, J. D., Rodríguez, C. G., González, P. B., Design aspects and two-dimensional CFD simulation of a marine propulsor based on a biologically-inspired undulating movement. Journal of Maritime Research, vol. 7, No. 2, pp. 7387, 2010.

11.Rodríguez, J., González, P., Couce, A., González, G., (2009) Diseño de sistema de propulsión ondulante. Proceedings of XIII International Congress on Project Engineering, June, Badajoz, Spain, pp. 1961-1972.

12.Versteeg H K, Malalasekera W. An introduction to computacional fluid dynamics: the finite volume method. $2^{\text {nd }}$ edition. Harlow: Pearson Education, 2007. 503p. ISBN 978-013-127498-3.

13.Fluent 6.3 Documentation, 2006, Fluent Inc.

14.Deng, J., Shao, X. M., Ren, A. L., (2005), Numerical study on propulsive performance of fish-like swimming foils, Journal of Hydrodynamics, Ser. B 18(6), pp. 681-687.

\section{CONTACT WITH THE AUTHORS}

Lamas M. I., Assoc. Prof.

Rodríguez J. D., Assoc. Prof.

Rodríguez C. G., M. Sc.

González P. B., Prof.

Escola Universitaria Politécnica. Universidade da Coruña. Avda. 19 de Febreiro s/n - 15405 Ferrol - A Coruña. Spain. e-mail: isabellamas@udc.es 